\title{
Apprentissage par projet en électrotechnique : optimisation d'une génératrice électrique à aimants permanents intégrée au moteur d'un avion
}

\author{
B. Dehez*, E. Matagne, V. Kluyskens \\ \{dehez, matagne, kluyskens\}@lei.ucl.ac.be \\ Département d'électricité (ELEC) \\ Université catholique de Louvain (UCL) \\ 3, Place du Levant, B-1348 Louvain-la-Neuve, Belgique
}

\begin{abstract}
RESUME
L'Université catholique de Louvain pratique depuis plusieurs années une pédagogie active dans les premières années de formation des ingénieurs civils. Forts de cette expérience, certains enseignants tentent d'appliquer ce type de pédagogie à un stade plus avancé de la formation. Dans ce contexte, les enseignants des cours de convertisseurs électromécaniques ont proposé, dans le cadre d'un cours plus avancé dans ce domaine, un projet portant sur l'optimisation d'une génératrice à aimants permanents pour une application en aéronautique. Le présent article présente ce projet, la manière dont il a été organisé ainsi que les principaux résultats techniques et pédagogiques qui ont été atteints.
\end{abstract}

Mots clés : Convertisseurs électromécaniques, génératrices à aimants permanents, modèle thermique, modèle électromagnétique, optimisation multivariable

\section{INTRODUCTION}

Le recours à la pédagogie active est une tendance qui s'observe dans tous les programmes de formation, des cycles les plus élémentaires aux plus avancés, aussi bien dans le domaine des sciences humaines que des sciences exactes.

Alors que les pédagogies traditionnelles sont centrées sur l'apprentissage des contenus au travers de cours magistraux, la pédagogie active implique davantage les étudiants dans leur formation. Typiquement, il s'agit d'introduire les contenus au travers de situations problèmes que l'étudiant devra résoudre seul, ou plus fréquemment, en groupe. Dans la pratique, l'acquisition des concepts de base se fait davantage via des exercices plus concis et plus ciblés alors que l'intégration et la contextualisation de ces concepts se fait plutôt au travers de projets d'une ampleur plus conséquente.

Depuis l'année académique 2000-2001, la Faculté des Sciences Appliquées de l'Université catholique de Louvain a recours à cette forme de pédagogie dans le cadre de la formation des bacheliers en science de l'ingénieur, orientation ingénieur civil. L'impact de ce changement radical dans la façon d'enseigner aux ingénieurs a fait l'objet d'une étude menée entre 2000 et 2004 par une équipe multidisciplinaire intégrant enseignants, étudiants, chercheurs en pédagogie universitaire et formateurs [1]. Sans entrer dans le détail de cette étude, il est intéressant de noter qu'elle a permis de montrer que, sans être pénalisés au niveau de leurs acquis «notionnels», les étudiants ont pu développer d'autres capacités, dont celle de travailler en groupe ou de résoudre un problème.

Au niveau des masters, cette forme de pédagogie n'a pas été appliquée de manière systématique. Le choix a été laissé aux titulaires de cours de conserver ou non une méthode d'enseignement plus traditionnelle. S'il faut constater que ces méthodes sont encore majoritaires dans le second cycle, l'expérience acquise en apprentissage actif par les professeurs impliqués dans le premier cycle est à l'origine d'une série d'initiatives pédagogiques allant dans ce sens.

C'est finalement le cas du projet que nous présentons dans cet article et que nous proposons aux étudiants ingénieurs civils de quatrième année qui entament un master en électromécanique. Ce projet s'intègre dans la formation en électrotechnique et plus spécifiquement au cours plus avancé traitant de la physique interne des convertisseurs électromécaniques [2].

\section{OBJECTIFS PÉDAGOGIQUES}

Le cours de physique interne des convertisseurs électromécaniques fait suite à un cours de base [3] durant lequel sont introduits les principes de la conversion électromécanique d'énergie, en particulier au sein des convertisseurs électromagnétiques.

En termes de compétences, l'objectif de ce cours est de donner aux étudiants les outils nécessaires à la modélisation des convertisseurs à couplage électromagnétique pour pouvoir ensuite en évaluer les performances et établir des méthodes de conception et d'optimisation de tels dispositifs.

Pour atteindre cet objectif, le dispositif mis en place se constitue de cours magistraux donnés durant les sept premières semaines du quadrimestre et d'un projet s'étalant sur les quatorze semaines que compte le quadrimestre.

Le cours magistral permet d'introduire de manière théorique les différents outils de modélisation: modèles de type champ et de type circuit, méthodes analytiques et numériques pour le calcul des champs, de l'énergie et des forces, méthodes 
d'homogénéisation, modélisation des matériaux magnétiques (pertes, non-linéarités, points de fonctionnement), ...

Le projet, réalisé par groupes de quatre étudiants, permet pour sa part de contextualiser et de manipuler les notions théoriques énoncées ci-dessus en développant le modèle électromagnétique d'un convertisseur. Il permet également d'aller plus loin puisqu'il est demandé de dimensionner et d'optimiser ce convertisseur pour une application spécifique. Pour y parvenir, le modèle électromagnétique doit donc être complété par différents modules physiques : un modèle thermique du convertisseur et de son système de refroidissement, un modèle de la charge, ...

A l'heure des outils de calcul numérique, ce projet montre l'intérêt que peut avoir un modèle analytique qui, construit sur base d'hypothèses bien choisies, permettra de rendre compte de la réalité tout en restant manipulable dans le cadre d'une optimisation. En effet, vu la complexité du système mis à l'étude et le nombre de paramètres en jeu, il n'est pas imaginable de recourir à des outils de calcul numérique pour mener une optimisation globale du système.

\section{ENONCÉ}

L'objectif du projet est d'optimiser une génératrice à aimants permanents en fonction d'une utilisation particulière, à savoir alimenter un avion de ligne en énergie électrique en prélevant de l'énergie mécanique disponible sur l'arbre de l'étage basse pression des moteurs à réaction, du côté compresseur (Fig. 1).

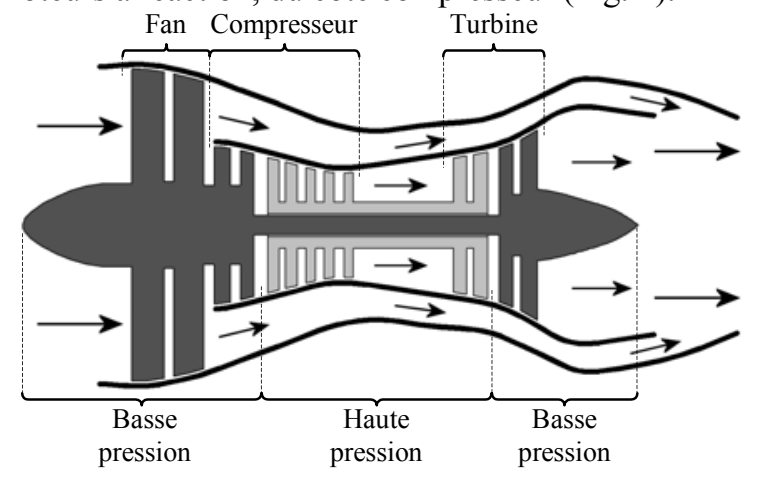

Fig. 1. Turboréacteur à double flux

Le type et la structure de la génératrice (Fig. 2) ont été imposés aux étudiants dès le début de projet en justifiant ce choix par rapport aux autres solutions envisageables.

Dans cette structure, le rotor, intérieur, est solidarisé à l'axe de l'étage basse pression. Le nombre de paires de pôles, le nombre d'encoches du stator et les différentes dimensions géométriques sont laissés libres et devront être déterminés lors de l'optimisation.

Pour être intégrée au turboréacteur, la génératrice doit entrer dans un volume ayant la forme d'un manchon d'une longueur de $213 \mathrm{~mm}$, d'un rayon extérieur de $181 \mathrm{~mm}$ et d'un rayon intérieur de $87 \mathrm{~mm}$.

$\mathrm{Au}$ niveau thermique, il faut garantir que la température à l'intérieur des enroulements ne dépasse pas $240{ }^{\circ} \mathrm{C}$ afin d'obtenir une durée de vie suffisante sans devoir utiliser des isolants minéraux.

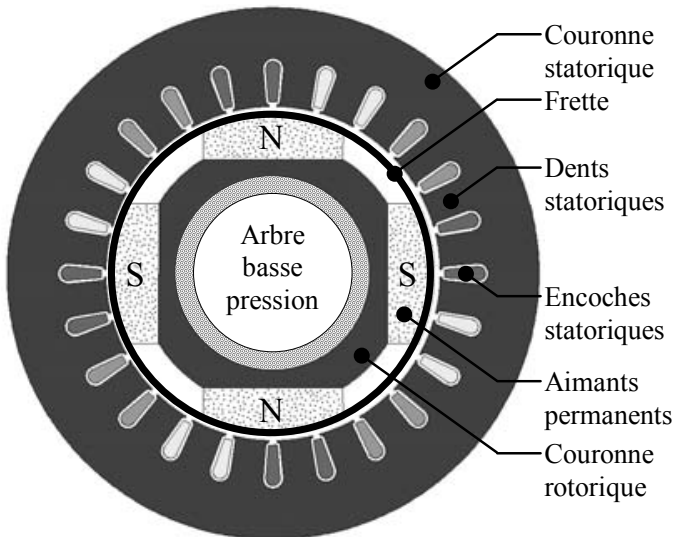

Fig. 2. Structure de la génératrice à aimants permanents

$\mathrm{Au}$ niveau mécanique, il faut prévoir un jeu mécanique de $1 \mathrm{~mm}$ minimum entre le rotor et le stator de la génératrice. A cela s'ajoute une frette de $1 \mathrm{~mm}$ d'épaisseur, non magnétique, destinée à protéger les aimants (Fig. 2).

L'ensemble de la génératrice est entouré d'une carcasse de $3 \mathrm{~mm}$ d'épaisseur. Entre celle-ci et le dos de la couronne statorique est disposé un circuit de refroidissement alimenté par de l'huile à $140{ }^{\circ} \mathrm{C}$.

L'énergie électrique produite est fournie à un réseau local à courant continu au travers d'un convertisseur électronique de puissance $\mathrm{AC} / \mathrm{DC}$ de type redresseur MLI dont la tension nominale côté alternatif est limitée à $250 V_{\text {eff. }}$ Ce redresseur est supposé pouvoir fournir à la génératrice un courant réactif. Les pertes de l'électronique sont estimées proportionnelles au courant efficace, et son rendement en régime nominal de $97 \%$ pour un facteur de puissance unitaire.

Le but de l'optimisation proprement dite est de maximiser la puissance fournie par la génératrice tant qu'une puissance de $100 \mathrm{~kW}$ n'est pas atteinte pour les différents régimes moteurs, c'est-à-dire de 1200 à $4500 \mathrm{rpm}$. Si cette puissance est atteinte, l'optimisation portera alors sur la masse de la génératrice.

\section{DÉMARCHE}

Afin d'aider les étudiants dans la résolution du problème proposé, nous avons scindé celui-ci en six phases détaillées ci-dessous. Elles s'étalent sur une ou deux semaines et suivent un même schéma au niveau de l'organisation.

Elles débutent par une séance de lancement durant laquelle sont distribuées les consignes pour la phase : objectifs, organisation pratique, guidance et instructions pour le rapport. Cette première séance permet d'amorcer la réflexion avec les étudiants et de s'assurer qu'ils savent par où commencer. Il ne s'agit pas d'expliquer comment résoudre le problème mais bien de montrer qu'ils ont en main tous les éléments nécessaires pour y parvenir. Suivant les cas, la phase de lancement peut être également l'occasion de présenter 
aux étudiants certains outils : logiciel d'optimisation, logiciel de calcul numérique, ...

S'ensuit une première période de quelques jours pendant laquelle les étudiants travaillent de manière autonome sur le projet. Ils retrouvent ensuite les tuteurs du projet dans le cadre d'une séance de consultance durant laquelle ils peuvent exposer les difficultés qu'ils rencontrent dans la résolution du problème.

Une deuxième période de travail individuel amène les étudiants à la fin de la phase. Celle-ci s'achève par une séance de débriefing durant laquelle l'un des groupes d'étudiants présente ses résultats. Cette présentation est l'occasion de remettre au même niveau les différents groupes mais aussi d'aborder, sur base de l'expérience acquise par les étudiants, des questions plus avancées sur la matière.

$\mathrm{Au}$ terme de chacune des phases, les étudiants remettent un rapport intermédiaire qui sera corrigé et commenté par les tuteurs.

\subsection{Phase I : Identification des paramètres}

L'objectif principal de cette première phase est de découvrir la machine sur laquelle porte l'étude. Pour ce faire, il est demandé aux étudiants d'établir une liste complète et structurée des paramètres caractérisant cette machine au niveau de :

- son comportement électromagnétique ;

- son comportement thermique ;

- son volume ;

- sa masse.

La séance de lancement permet d'aider les étudiants à se poser les bonnes questions : Est-il pertinent de paramétrer le moindre détail de la machine ? Tous les paramètres proposés sont-ils indépendants ou certains peuvent-ils s'exprimer en fonction d'autres ?

Durant la séance de consultance les étudiants ont la possibilité de voir une maquette démontée de machine synchrone à aimants permanents. Cela leur permet de manipuler du concret, et notamment, de se rendre compte que les têtes de bobines présentent un encombrement tel qu'il devra être pris en compte dans l'expression du volume et de la masse de la machine. Les étudiants en profitent également pour prendre les mesures de la machine. Celles-ci leur serviront plus tard pour tester et valider leurs modèles sur base de données réalistes.

Le résultat de cette phase, une liste structurée des paramètres de la machine, est un véritable outil qui servira dans les différentes phases du projet, entre autres pour assurer une certaine cohérence dans les notations utilisées dans les différents modèles. A ce stade du projet, il est vraisemblable que la liste proposée par les étudiants soit incomplète. Elle devra donc évoluer au fur et à mesure de leur avancement dans le projet.

\subsection{Phase II : Modèle thermique}

L'objectif de la deuxième phase est de développer un modèle thermique analytique de la génératrice. En effet, celle-ci étant le siège de pertes, il faudra s'assurer, durant la phase d'optimisation, que les niveaux de température atteints dans les différentes parties de la machine ne risquent pas de l'endommager.

Pour simplifier l'élaboration du modèle, plusieurs hypothèses simplificatrices sont proposées d'emblée :

- Le régime thermique est établi.

- Les pertes se décomposent en pertes Joule dans les conducteurs statoriques et en pertes magnétiques (par hystérésis et par courants de Foucault) dans la couronne et les dents statoriques. Les pertes magnétiques au rotor, ainsi que les pertes mécaniques (i. e. pertes par frottement aérodynamique dans l'entrefer), sont négligées.

- La chaleur produite dans les têtes de bobine se répartit instantanément sur toute la longueur de la spire.

La méthode suggérée pour développer le modèle thermique est celle des circuits thermiques équivalents. La phase de lancement est alors l'occasion d'un rappel des notions de base en thermique ainsi que d'une introduction au logiciel de calcul numérique COMSOL Multiphysics $^{\circledR}$ qui doit non seulement aider les étudiants dans la construction de leur schéma thermique équivalent mais aussi leur permettre de valider leur modèle thermique.

Pour le système de refroidissement situé entre la carcasse de la machine et la couronne statorique, nous fournissons directement aux étudiants son modèle circuit : une référence de tension, d'une valeur égale à la température d'entrée de l'huile de refroidissement, reliée à une résistance thermique $R_{t h}$ fonction de la longueur du dispositif de refroidissement en contact avec la couronne statorique, de son épaisseur, et de son rayon moyen.

La première étape pour les étudiants a donc été de modéliser à l'aide du logiciel de calcul numérique la structure réduite, par symétrie, à une encoche et deux demi-dents. Ils ont pu de la sorte observer la forme des lignes de flux thermique et trouver le circuit thermique équivalent y correspondant au mieux (Fig. 3). A noter que les flux thermiques au travers de l'entrefer ont été, à juste titre, négligés dans le modèle de type circuit.

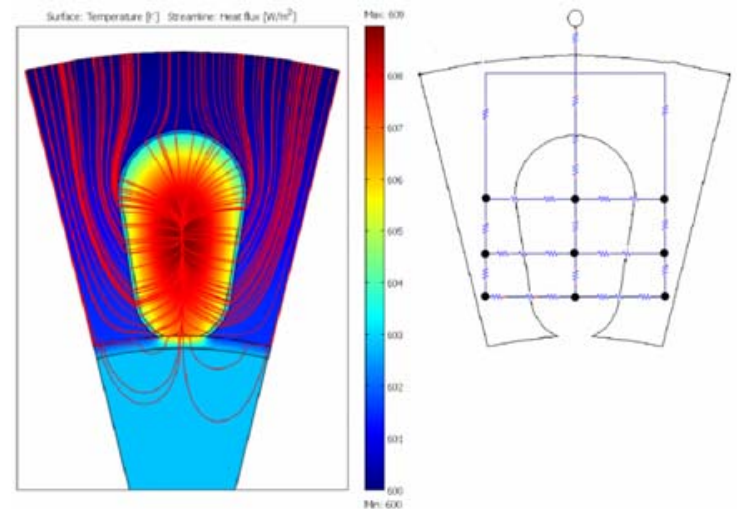

Fig. 3. Lignes de flux thermique et schéma thermique équivalent d'une encoche 
Les pertes par effet Joule dans les bobinages ainsi que les pertes magnétiques dans la couronne et les dents statoriques ont été introduites dans le schéma thermique équivalent via des sources de courants au niveau de certains nœuds (points noirs dans la Fig. 3).

Les propriétés des bobinages, et en particulier leur conductivité thermique équivalente, ont été obtenues par homogénéisation sur base de la formule [4] :

$$
\lambda_{e q}=\left[\left(1+\alpha \frac{\lambda_{1}-\lambda_{2}}{\lambda_{1}+\lambda_{2}}\right) /\left(1-\alpha \frac{\lambda_{1}-\lambda_{2}}{\lambda_{1}+\lambda_{2}}\right)\right] \lambda_{2}
$$

où $\lambda_{e q}$ est la propriété équivalente de deux matériaux caractérisés par les propriétés $\lambda_{1}$ et $\lambda_{2}$ et répartis suivant une proportion $\alpha$.

$\mathrm{La}$ résolution $\mathrm{du}$ schéma thermique équivalent permet alors d'exprimer la température aux différents nœuds en fonction des résistances et des sources thermiques. La comparaison entre les résultats de ce modèle analytique et les températures prédites par le logiciel de calcul numérique montre des erreurs de l'ordre de $20 \%$, ce qui, dans le cadre de ce projet, semble raisonnable.

\subsection{Phase III : Modèle électromagnétique simplifié}

L'objectif de la troisième phase est de développer un modèle permettant d'estimer la puissance électrique produite en fonction des paramètres de la génératrice et du régime moteur.

Pour faciliter le développement de ce modèle, la perméabilité magnétique des couronnes statorique et rotorique est supposée, pour cette phase, infinie. D'autres hypothèses simplificatrices sont également proposées :

- Seule la fondamentale de la composante azimutale du champ magnétique d'entrefer sera considérée (hypothèse du premier harmonique).

- La composante radiale du champ d'entrefer est constante sur toute l'épaisseur de l'entrefer. Cette hypothèse n'est cependant acceptable que si l'épaisseur effective de l'entrefer (frette et aimants compris) est petite à la fois par rapport au rayon de l'entrefer et par rapport au pas polaire.

\subsubsection{Champ d'entrefer dû aux aimants}

Sur cette base, les étudiants ont d'abord déterminé le champ d'entrefer généré par les aimants permanents $H_{e, a i m}$ en calculant leur point de fonctionnement. Considérant des aimants terre rare, dont la caractéristique magnétique est une droite, ce calcul trouve une solution analytique du type :

$$
B_{a}=\frac{e_{a}}{e^{\prime}} B_{r}
$$

où $B_{a}$ est l'induction magnétique de l'aimant au point de fonctionnement, $B_{r}$, son induction rémanente, $e_{a}$, son épaisseur, et $e$ ', l'épaisseur de l'entrefer équivalent lisse calculé par une méthode de type Carter [5].
La forme du champ d'entrefer suit alors une forme en créneau (Fig. 4) dont il est possible d'extraire la fondamentale :

$$
H_{e, a i m}(\theta)=\frac{4}{\pi} \frac{B_{a}}{\mu_{0}} \cos \left(\frac{p \beta}{2}\right) \cos \left(p\left(\theta-\omega_{m} t\right)\right)
$$

où $p$ est le nombre de paires de pôles de la machine, $\beta$, l'angle séparant deux aimants, $\theta$, la position le long de l'entrefer, $\omega_{m}$, la vitesse de rotation, et $t$, le temps.

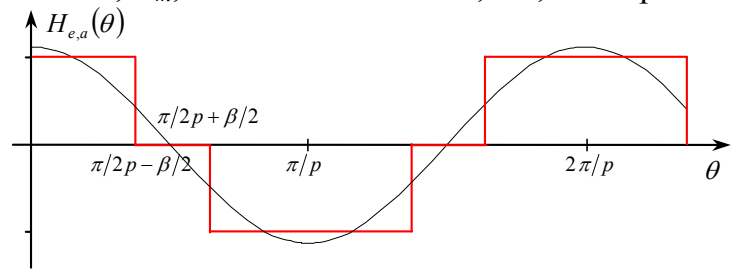

Fig. 4. Champ d'entrefer dî aux aimants

\subsubsection{Champ d'entrefer dû aux bobinages}

Pour ensuite déterminer le champ d'entrefer généré par les bobinages, les étudiants sont partis de l'expression du champ d'entrefer généré par un enroulement comportant $n$ spires et occupant $m$ paires d'encoches successives sous chaque paire de pôles :

$$
\begin{aligned}
H_{e, b o b, k}(\theta)=\frac{2 n}{\pi p e^{\prime}} \sum_{j=1}^{\infty}(-1)^{j} \frac{\sin ((2 j-1) \pi / 6)}{m \sin ((2 j-1) \pi / 6 m)} \\
\frac{\cos \left((2 j-1) p\left(\theta-\theta_{k}\right)\right)}{(2 j-1)} i
\end{aligned}
$$

où $k$ est l'indice de l'enroulement, $\theta_{k}$, la position de son axe magnétique, et $i$ le courant y circulant. Le champ total $H_{e, b o b}$ est alors obtenu par superposition des champs générés par le système triphasé d'enroulements statoriques. L'expression de sa fondamentale prend la forme :

$$
\begin{aligned}
H_{e, b o b}(\theta)=\frac{3 n \sqrt{2} \hat{I}_{s}}{\pi p e^{\prime}} \frac{\sin (\pi / 6)}{m \sin (\pi / 6 m)} \\
\cos \left(\omega_{s} t-p \theta+\varphi_{s}\right)
\end{aligned}
$$

où $\omega_{s}$ est la pulsation des courants statoriques, $\hat{I}_{s}$, leur valeur efficace, et $\varphi_{s}$, leur phase.

A noter que dans le cas d'une génératrice synchrone, $\omega_{s}$ et $\omega_{m}$ sont liés par la relation :

$$
\omega_{s}=p \omega_{m} .
$$

\subsubsection{Flux magnétique et FEM dans les bobinages}

Le calcul du flux dans les bobinages passe d'abord par celui du champ total d'entrefer $H_{e, t o t}$, somme du champ dû aux aimants et du champ dû aux bobinages. Ces deux champs étant déphasés spatialement, le plus simple pour se représenter cette somme est de passer par un diagramme phasoriel sur lequel il est déjà possible de tracer les phaseurs des courants statoriques $I_{s}$, en phase avec le champ d'entrefer qu'ils génèrent, du flux total $\Phi_{t o t}$, en phase avec le champ d'entrefer total, et de la force électromotrice $E$, en quadrature avant avec le flux total (Fig. 5). 


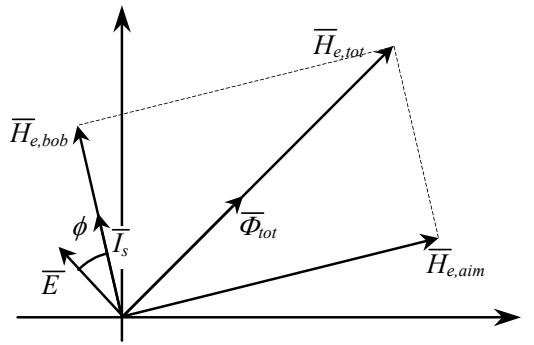

Fig. 5. Diagramme phasoriel

Une analyse de ce diagramme phasoriel montre qu'un déphasage de $90^{\circ}$ entre les champs dus aux aimants et aux bobinages maximise la puissance active, produit de la force électromotrice et du courant, fournie par la génératrice. Or ce déphasage peut être imposé par l'électronique de puissance connectée à la sortie de la génératrice via le contrôle du courant réactif qu'elle fournit.

Sur cette base, l'expression du champ total d'entrefer prend la forme :

$$
\begin{aligned}
H_{e, t o t}(\theta)=\sqrt{2} \sqrt{\hat{H}_{e, a i m}^{2}+\hat{H}_{e, b o b}^{2}} & \cos \left(p\left(\omega_{m} t-\theta\right)-\psi\right)
\end{aligned}
$$

où $\hat{H}_{e, a i m}$ et $\hat{H}_{e, b o b}$ sont les valeurs efficaces des champs d'entrefer dus, respectivement, aux aimants et aux bobinages, et $\psi$, un déphasage qui disparaîtra par la suite.

Connaissant le champ d'entrefer total, le flux dans une phase s'obtient via l'intégrale :

$$
\Phi_{t o t}=n \int_{-\pi / 2 p}^{\pi / 2 p} \mu_{0} H_{e, t o t}(\theta) L R d \theta
$$

où $R$ est le rayon moyen de l'entrefer. Par dérivation, ce dernier donne alors la force électromotrice :

$$
\begin{gathered}
E=2 \sqrt{2} n L R \mu_{0} \hat{H}_{e, t o t} \omega_{m} \\
\frac{\sin (\pi / 6)}{m \sin (\pi / 6 m)} \sin \left(p \omega_{m} t-\psi\right)
\end{gathered}
$$

en introduisant au passage le coefficient de filtrage lié à l'étalement des bobinages.

\subsubsection{Puissance}

La puissance fournie par la génératrice sur une phase est donnée par le produit de la force électromotrice et du courant sur cette phase. En se référant au diagramme phasoriel (Fig. 5), elle peut s'exprimer comme suit :

$$
P_{1 \varphi}=\hat{E} \hat{I}_{s} \cos \phi
$$

où $\hat{E}$ est la valeur efficace de la force électromotrice.

Il est également possible d'observer que seule la force électromotrice due au champ d'entrefer généré par les aimants entre en jeu dans l'expression de la puissance. On peut aussi comprendre l'intérêt d'imposer un déphasage de $90^{\circ}$ entre le champ d'entrefer dû aux aimants et aux courants circulants dans les bobinages en vue de maximiser la puissance active. Dans ce cas particulier, la puissance triphasée, objectif de cette troisième phase, prend finalement la forme :

$$
P_{3 \varphi}=6 n L R \mu_{0} \hat{H}_{e, a i m} \hat{I}_{s} \omega_{m} \frac{\sin (\pi / 6)}{m \sin (\pi / 6 m)} .
$$

\subsection{Phase IV : Modèle électromagnétique avancé}

Pour développer le modèle électromagnétique simplifié, les étudiants ont supposé la perméabilité magnétique des matériaux ferromagnétiques infinie. Dans cette quatrième phase, nous leur avons demandé de considérer le cas où cette perméabilité est non seulement finie mais en plus dépendante du niveau de magnétisation de la machine.

La séance de lancement est dans ce cas-ci l'occasion d'expliquer aux étudiants l'intérêt de prendre en compte des propriétés réelles des matériaux magnétiques dans le processus d'optimisation de la machine, à savoir :

- prendre en compte l'influence des dimensions des circuits magnétiques rotorique et statorique sur la force électromotrice induite et la puissance ;

- déterminer les niveaux d'induction magnétique nécessaires au calcul des pertes magnétiques.

\subsubsection{Méthode de calcul}

La perméabilité finie des matériaux ferromagnétiques de la machine peut être prise en compte en appliquant un principe de superposition.

En effet, contrairement au cas d'une perméabilité infinie, les champs magnétiques ne peuvent plus être considérés comme nuls dans les parties ferromagnétiques de la machine. A ces champs, $H_{f e r, s}$ au stator et $H_{f e r, r}$ au rotor, correspond donc une circulation magnétique à laquelle il est possible d'associer une différence de potentiel magnétique, $\Delta \Theta_{s}$ pour le stator et $\Delta \Theta_{r}$ pour le rotor (Fig. 6). Il résulte donc de ces champs une différence de potentiel magnétique entre le stator et le rotor, $\Delta \Theta_{s-r}$, à l'origine d'un flux magnétique d'entrefer $\Delta \Phi$ qui vient se superposer et corriger le flux magnétique $\Phi_{t o t}$ calculé pour une perméabilité magnétique infinie.

Concrètement, le point de départ pour le calcul de la correction est le champ magnétique d'entrefer $H_{e, t o t}$ calculé lors de la phase précédente. Intégré sur la largeur d'un pas dentaire (distance entre deux encoches), il donne les flux verticaux $\Phi_{V}$ traversant les dents statoriques et leurs vis-à-vis rotoriques. Des flux verticaux, il est possible de déterminer les flux horizontaux passant d'une dent à l'autre au niveau des couronnes statoriques, $\Phi_{H, s}$, et rotorique, $\Phi_{H, r}$. Connaissant ces flux, il est alors facile de calculer, sur base des dimensions des couronnes et des dents, les champs magnétiques dans les différentes parties des circuits magnétiques statorique et rotoriques. En reprenant le raisonnement présenté ci-dessus, on obtient alors le flux corrigé. Pour être tout à fait correct, il faut reproduire l'ensemble des calculs sur 
base des nouveaux flux, et ce, jusqu'à convergence. En pratique, les étudiants ont pu observer que cette convergence était atteinte en seulement trois ou quatre itérations.

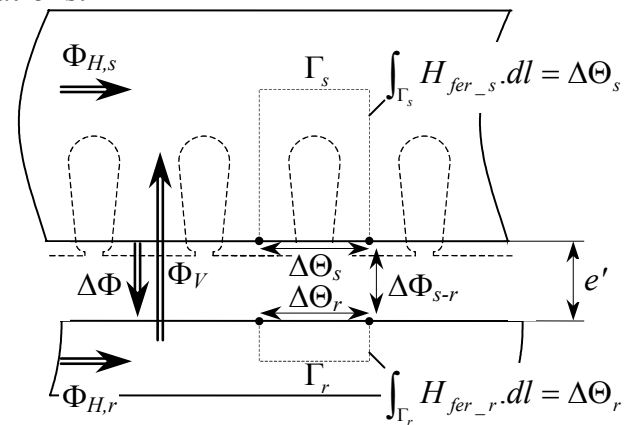

Fig. 6. Illustration des principaux paramètres intervenant dans le calcul du modèle électromagnétique avancé

Dans la démarche proposée ci-dessus, la saturation peut être prise en compte en exprimant le fait que la perméabilité magnétique n'est plus une constante mais bien une fonction de l'induction magnétique. Cela influence d'abord le calcul des flux horizontaux sur base des flux verticaux et ensuite celui des champs magnétiques sur base des flux verticaux et horizontaux.

\subsubsection{Puissance corrigée}

Connaissant le flux corrigé, la force électromotrice et la puissance active fournie par la machine peuvent être déduites successivement en suivant les mêmes développements que ceux présentés dans la phase III.

\subsubsection{Pertes magnétiques}

Les champs magnétiques dans la couronne et les dents statoriques apparaissent comme variables intermédiaires dans le calcul du flux corrigé. Ils peuvent donc être récupérés pour déterminer le niveau de pertes magnétiques dans ces différentes zones. Ces pertes sont données par la formule suivante :

$$
P_{\text {pertes }}=\left(k_{\text {hyst }} f_{s} B_{m}^{2}+k_{\text {Fouc }} f_{s}^{2} B_{m}^{2}\right) \rho V
$$

où $k_{\text {hyst }}$ et $k_{\text {Fouc }}$ sont des coefficients empiriques propres aux matériaux utilisés et caractérisant les niveaux de pertes par hystérésis et par courant de Foucault respectivement, $f_{s}$ est la fréquence électrique, directement liées à la vitesse de rotation par la relation (6), $B_{m}$ est l'amplitude de l'induction magnétique, $\rho$ est la densité du matériau considéré et $V$ son volume.

\subsection{Phase $V$ : Validation électromagnétique}

Afin de valider leur modèle électromagnétique, les étudiants ont pu réaliser une série de mesures sur la maquette présentée lors de la première phase du projet. Grâce à un moteur DC utilisé pour entraîner cette machine synchrone, ils ont notamment pu relever :

- l'amplitude et la forme de la tension de sortie à vide ;

- le niveau de pertes magnétiques ;

pour ensuite les comparer aux prédictions de leur modèle. Ils ont ainsi observé les limites de ce modèle et de certaines des hypothèses introduites pour le développer.

Cette validation expérimentale aurait pu être complétée, voire remplacée, par une validation menée sur base d'une modélisation numérique de la machine. C'est une voie que nous souhaiterions suivre dans les prochaines années car elle répondrait mieux à nos objectifs pédagogiques. Cependant, pour éviter que les étudiants passent plus de temps à apprendre un logiciel qu'à exploiter les résultats qu'il fournit, cette voie nécessitera une bonne préparation de la part de l'équipe enseignante.

\subsection{Phase VI : Optimisation}

L'objectif de cette dernière phase est d'exploiter, au sein d'un logiciel d'optimisation spécifique, les différents modules physiques qui ont été développés dans le cadre du projet et qui interviennent dans le modèle global du système.

Le logiciel d'optimisation utilisé dans le cadre de ce projet est le logiciel Pro@DESIGN ${ }^{\circledR}$ [6]. Présenté aux étudiants en début de projet, il offre comme principal avantage de ne pas demander de programmation à proprement parler puisqu'il suffit de lui fournir les équations analytiques du modèle. Il se charge ensuite de les analyser avant de proposer un environnement d'optimisation où les paramètres du modèle, paramètres d'entrée (variables indépendantes) et paramètres de sortie (variables dépendantes) sont accessibles soit :

- pour les proposer comme fonction objectif ;

- pour leur imposer une valeur initiale ;

- pour les contraindre dans un intervalle de valeurs ;

- ...

Cette dernière phase n'a pas été sans poser quelques difficultés aux étudiants. Tout d'abord, les modèles développés en cours de projet n'étaient pas tous exploitables tel quel dans le logiciel d'optimisation. Pour exemple, dans le modèle thermique de la phase II, les étudiants s'étaient limités à exprimer sous forme matricielle les relations entre les résistances thermiques, les sources de chaleur et les températures aux différents nœuds du circuit thermique équivalent. Le logiciel d'optimisation n'acceptant pas ce type de formalisme, il leur a donc fallu résoudre symboliquement le système d'équation pour dégager une expression explicite de la température en fonction des paramètres de la machine.

Ensuite, plusieurs modèles, construits sur base d'une valeur prédéfinie de certains paramètres de la machine, n'étaient donc pas adaptés à une optimisation multivariable. Dans le cas du modèle électromagnétique de la phase IV, il était par exemple indispensable de fixer a priori le nombre de dents par paires de pôles, autrement dit la valeur $m$ de l'étalement, pour pouvoir résoudre le problème. La solution retenue par les étudiants a été de considérer des enroulements concentrés sur une seule encoche 
pour le calcul des flux, mais une largeur de dent multipliée par la valeur de l'étalement $m$ pour le calcul des champs.

Enfin, les étudiants ont dû apprendre à gérer les boucles implicites apparaissant au sein de leurs différents modèles. L'exemple le plus parlant est celui du calcul du flux magnétique corrigé dans la phase IV. Telle que présentée ci-dessus, la solution est obtenue par une itération de la forme :

$$
\left\{\begin{array}{l}
\Phi_{c o r r, i}=\Phi_{t o t}+\Delta \Phi_{i} \\
\Delta \Phi_{i}=f\left(\Phi_{c o r r, i-1}\right)
\end{array}\right.
$$

où $\Phi_{c o r r, i}$, le flux corrigé à l'itération $i$, est posé égale à $\Phi_{\text {tot }}$ pour la première itération. Ce mode de résolution cache en fait une boucle implicite du type :

$$
\left\{\begin{array}{l}
\Phi_{c o r r}=\Phi_{t o t}+\Delta \Phi \\
\Delta \Phi=f\left(\Phi_{c o r r}\right)
\end{array},\right.
$$

que les étudiants sont parvenus à expliciter, mais qui ne peut être prise en compte tel quel dans le logiciel d'optimisation. Plusieurs solutions sont alors envisageables, la plus efficace étant de résoudre analytiquement cette boucle pour exprimer le flux corrigé $\Phi_{\text {corr }}$ directement comme une fonction du flux $\Phi_{t o t}$ calculé pour une perméabilité magnétique infinie. Les autres solutions passent par le logiciel d'optimisation lui-même. Elles se justifient dans le cas où les boucles implicites ne peuvent être résolues analytiquement, ce qui est notamment le cas lorsque la saturation magnétique est prise en compte dans le modèle magnétique. L'une d'elles consiste à ouvrir la boucle implicite via un paramètre supplémentaire introduit dans le modèle et à imposer sa fermeture en contraignant ce paramètre à s'annuler dans l'optimisation. Dans le cas qui nous intéresse, ce paramètre supplémentaire pourrait être :

$$
c=f\left(\Phi_{\text {corr }}\right)-\Delta \Phi,
$$

Une fois les différents modèles intégrés dans le logiciel d'optimisation, les étudiants ont du définir les contraintes et trouver les conditions initiales assurant au logiciel une convergence vers une solution satisfaisante.

$\mathrm{Au}$ final, les étudiants sont parvenus à exploiter le logiciel d'optimisation pour dimensionner une génératrice capable de fournir une puissance de $100 \mathrm{~kW}$ aux différents régimes moteur de l'avion tout en respectant les contraintes de température et d'encombrement.

\section{CONCLUSION}

Les nombreux concepts introduits dans le cadre des cours portant sur les convertisseurs électromécaniques sont parfois difficiles à s'approprier, et ce, même s'ils sont souvent illustrés au travers de séances d'exercices ou de laboratoires.

L'idée d'intégrer un projet d'ampleur dans un cours plus avancé consacré à cette matière avait pour principal objectif de placer les étudiants dans une position inédite où, à partir d'un contexte donné, ils doivent pouvoir décider des concepts à mettre en application pour élaborer une solution. Plus spécifiq uement, nous souhaitions que les étudiants prennent pleinement conscience de l'intérêt d'un modèle analytique bien construit lorsqu'il s'agit de concevoir et d'optimiser un convertisseur électromécanique.

Arrivé au terme de ce projet, nous estimons avoir pleinement atteint ces objectifs. Les étudiants semblent s'être effectivement appropriés de manière beaucoup plus forte les concepts appliqués dans le cadre du projet, tout en ayant acquis une approche plus mature du domaine dans son ensemble. En outre, au vu des difficultés rencontrées lors de la phase d'optimisation, nous estimons également qu'ils ont bien compris l'intérêt de développer des modèles analytiques à la fois simples, pour rester manipulables, et évolués, pour rendre compte de la réalité.

Au niveau de la démarche, nous avons pris le parti de subdiviser le projet en six phases échelonnées sur les quatorze semaines de cours. L'objectif poursuivi était non seulement d'aider les étudiants dans la résolution du problème, mais aussi d'éviter qu'ils ne concentrent leur travail en fin de période. L'organisation des phases suivant le schéma - séance de lancement, séance de consultance et séance de débriefing - est également de nature à aider les étudiants dans la construction d'une solution aussi avancée que possible à chacune des phases du projet.

Plus spécifiquement, la séance de débriefing, organisée autour de la présentation de l'un des groupes d'étudiants et clôturée par la remise d'un rapport, permet à la fois de stimuler les étudiants dans leur travail, mais aussi de les amener plus loin dans leurs réflexions sur le problème et la matière.

$\mathrm{Au}$ final, cette expérience pédagogique a été un réel succès, tant du point de vue des enseignants que des étudiants. Très motivés par le projet et la démarche suivie, ils n'ont pas hésité à investir beaucoup de leur temps, plus de 120 heures pour certains groupes.

\section{RÉFÉRENCES}

[1] B. Galand et M. Frenay, L'approche par problèmes et par projets dans l'enseignement supérieur: Impacts, enjeux et défis, Presses universitaires de Louvain, 2005.

[2] http://www.ucl.ac.beletudes/2007/cours/fr/

[3] http://www.ucl.ac.beletudes/2007/cours/fr/ elec2311.pdf elec1310.pdf

[4] E. Matagne, «Modélisation macroscopique des faisceaux de conducteurs », Journal de Physique III, mai 1993, pp. 509-517.

[5] http://www.lei.ucl.ac.be/ matagne/ELEC2311/

INDEX.HTM

[6] http://www.designprocessing.com/ 